\title{
Mechanistic Insights into Diblock Copolymer Nanoparticle-Crystal Interactions Revealed via in Situ Atomic Force Microscopy
}

DOI:

10.1021/jacs.8b03828

\section{Document Version \\ Accepted author manuscript}

Link to publication record in Manchester Research Explorer

Citation for published version (APA):

Hendley IV, C. T., Fielding, L. A., Jones, E. R., Ryan, A. J., Armes, S. P., \& Estroff, L. A. (2018). Mechanistic Insights into Diblock Copolymer Nanoparticle-Crystal Interactions Revealed via in Situ Atomic Force Microscopy. Journal of the American Chemical Society, 140(25), 7936-7945. https://doi.org/10.1021/jacs.8b03828

\section{Published in:}

Journal of the American Chemical Society

\section{Citing this paper}

Please note that where the full-text provided on Manchester Research Explorer is the Author Accepted Manuscript or Proof version this may differ from the final Published version. If citing, it is advised that you check and use the publisher's definitive version.

\section{General rights}

Copyright and moral rights for the publications made accessible in the Research Explorer are retained by the authors and/or other copyright owners and it is a condition of accessing publications that users recognise and abide by the legal requirements associated with these rights.

\section{Takedown policy}

If you believe that this document breaches copyright please refer to the University of Manchester's Takedown Procedures [http://man.ac.uk/04Y6Bo] or contact uml.scholarlycommunications@manchester.ac.uk providing relevant details, so we can investigate your claim.

\section{OPEN ACCESS}




\section{MECHANISTIC INSIGHTS INTO DIBLOCK COPOLYMER NANOPARTICLE-CRYSTAL}

INTERACTIONS REVEALED VIA IN SITU ATOMIC FORCE MICROSCOPY

Coit T. Hendley IV ${ }^{1, \#}$, Lee A. Fielding ${ }^{2}$, Elizabeth R. Jones ${ }^{3}$, Anthony Ryan ${ }^{3}$, Steven P. Armes ${ }^{3}$, Lara A. Estroff $f^{1,4 *}$

${ }^{1}$ Department of Materials Science and Engineering, Cornell University, Ithaca, NY 14853, USA.

${ }^{2}$ The School of Materials, University of Manchester, Oxford Road, Manchester, M13 9PL, UK

${ }^{3}$ Department of Chemistry, University of Sheffield, Brook Hill, Sheffield, Yorkshire, S3 7HF, UK.

${ }^{4}$ Kavli Institute at Cornell for Nanoscale Science, Cornell University, Ithaca, NY 14853, USA.

\# Current Address: C.T.H.: Naval Surface Warfare Center, Indian Head, MD 20640, USA.

*Corresponding author can be contacted at: lae37@cornell.edu 


\section{Abstract}

Recently, it has become clear that a range of nanoparticles can be occluded within single crystals to form nanocomposites. Calcite is a much-studied model, but even in this case we have yet to fully understand the details of the nanoscale interactions at the organic-inorganic interface that lead to occlusion. Here, a series of diblock copolymer nanoparticles with well-defined surface chemistries were visualized interacting with a growing calcite surface using in situ Atomic Force Microscopy (AFM). These nanoparticles comprise a poly(benzyl methacrylate) (PBzMA) core-forming block and either a non-ionic poly(glycerol monomethacrylate) (PhPGMA), a carboxylic acid-tipped poly(glycerol monomethacrylate) (HOOC-PGMA), or an anionic poly(methacrylic acid) (PMAA) as the stabilizer block. Our results reveal three modes of interaction between the nanoparticles and the calcite surface: (i) attachment followed by detachment, (ii) sticking to and "hovering" over the surface, allowing steps to pass beneath the immobilized nanoparticle, and (iii) incorporation of the nanoparticle by the growing crystals. By analyzing the relative contributions of these three types of interactions as a function of nanoparticle surface chemistry, we show that $\sim 85 \%$ of $\mathrm{PMAA}_{85}-\mathrm{PBzMA}_{100}$ nanoparticles either "hover" or become incorporated, compared to $~ 50 \%$ of the HOOC-PGMA71-PBzMA 100 nanoparticles. To explain this difference, we propose a two-state binding mechanism for the anionic PMAA $85-P_{8 z M A} 100$ nanoparticles. The "hovering" nanoparticles possess highly extended polyelectrolytic stabilizer chains and such chains must adopt a more "collapsed" conformation prior to successful nanoparticle occlusion. This study provides a conceptual framework for understanding how sterically-stabilized nanoparticles interact with growing crystals, and suggests design principles for improving occlusion efficiencies. 


\section{Introduction}

Biomineralization provides countless examples of the complexity that is accessible by manipulating crystallization via additives such as small molecules or proteins. ${ }^{1,2}$ The structural diversity among biominerals is in part due to biomacromolecules, which affect the nucleation and growth of the crystals and can also become incorporated within the crystals. ${ }^{2,3}$ The mechanisms by which atomic and molecular additives interact with the growing crystal are relatively well understood. ${ }^{4-10}$ Incorporation of larger organic additives, however, is less well studied, with only a handful of in situ Atomic Force Microscopy (AFM) investigations of peptides, proteins, and synthetic anionic block copolymer nanoparticles. ${ }^{11-15}$ In particular, in situ studies have demonstrated that additive-crystal interactions with multivalent additives are complex, often involving a multistep process through a weak and strong binding state. ${ }^{16,17}$ Thus a better understanding of the details of additive-crystal interactions is required to guide the rational synthesis of nanoscale additives for occlusion within single crystals in order to modify their properties. In this study, in situ AFM is used to analyze the role of surface chemistry in driving the interactions between diblock copolymer nanoparticles ( $>10 \mathrm{~nm}$ diameter) and growing calcite $\left(\mathrm{CaCO}_{3}\right)$ crystals.

One key aspect of additive-crystal interactions is that the occlusion mechanism depends on the additive size. Single atoms, small molecules (e.g., amino acids), and proteins are all found within calcitic biominerals and affect crystal growth differently. ${ }^{4,6,18,19}$ For example, $\mathrm{Mg}^{2+}$ is readily incorporated into calcite by substituting for $\mathrm{Ca}^{2+}$ cations in the crystal lattice. ${ }^{6,20}$ In the case of small molecules, the interaction with calcite is more complex, with chemical functionality determining the preference for binding to crystal surfaces at kinks and growing step 
edges. $^{5,7}$ For example, glycine and aspartic acid preferentially bind to acute step edges of calcite growth hillocks via their carboxylic acid groups. ${ }^{5}$ Additionally, molecules that cannot easily fit into the crystal structure must be accommodated by larger vacancies within the crystal. ${ }^{4}$ For larger molecules, i.e. peptides or polymers, length scales become important because the number of potential bonding units in a chain impacts the ability of such macromolecules to adsorb at a step edge. For example, a study using synthetic peptides showed that their potency of inhibition increases significantly for longer chains. ${ }^{15}$ The dynamics of binding and crystal growth are even more complex for synthetic polymers; some polymers can actually enhance crystal growth rates when present at very low concentration. ${ }^{15,21}$ At larger length scales, globular proteins and nanoparticles interact with a much larger surface area of the crystal, and as such the surface chemistry of such additives is expected to play a critical role in determining the mechanism of interaction with a growing crystal. For nanoparticles, very little is known about the design rules for efficient occlusion other than the importance of anionic character and chemical functionality of the stabilizer chains. ${ }^{22-24}$

Based on extensive research over the past ten years, the key factor driving nanoparticle incorporation into calcite is the surface chemistry and charge density of the anionic steric stabilizer chains. Particle incorporation was first demonstrated by Wegner and co-workers, who developed various anionic latexes that became occluded within calcite and $\mathrm{ZnO}$ single crystals. ${ }^{25,26}$ More recently, Meldrum and co-workers demonstrated that polystyrene latexes with polycarboxylated stabilizer chains could also be incorporated into calcite, while particles with

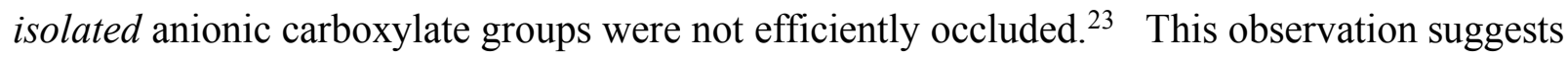
that cooperativity is important for binding to the calcite lattice. The same team, working closely with various other groups, subsequently showed that micellar diblock copolymer nanoparticles of 
$\sim 15 \mathrm{~nm}$ diameter comprising an anionic poly(2-(succinyloxy)propyl methacrylate) (PSPMA) stabilizer block could also be incorporated into calcite crystals. ${ }^{24,27}$ Recent progress has been made towards an empirical understanding of "design rules" for optimizing organic nanoparticle incorporation into calcite. ${ }^{22}$ In particular, it appears that the relative surface density of anionic stabilizer chains can determine whether or not nanoparticle occlusion is observed. ${ }^{28}$ Nevertheless, a more detailed understanding of the role of surface chemistry in determining nanoparticle occlusion mechanism(s) should inform the rational design of next-generation nanoparticles. In this context, direct observation of the nanoparticle-crystal interaction mechanism requires a high resolution in situ technique such as AFM.

A recent in situ AFM study by De Yoreo and co-workers revealed the mechanisms by which individual carboxylated PSPMA-stabilized diblock copolymer micellar nanoparticles are incorporated into calcite. ${ }^{12} \mathrm{~A}$ series of experiments demonstrated that these anionic copolymer nanoparticles strongly interact with calcite growth hillocks, bind to step edges, and quickly become engulfed under calcite growth conditions. Moreover, the occlusion mechanism appears to differ depending on the nanoparticle dimensions. ${ }^{12}$ Particles of $\sim 3 \mathrm{~nm}$ diameter are incorporated as spheres within the crystal lattice, but larger particles of $\sim 15 \mathrm{~nm}$ diameter are compressed into a cylindrical shape, apparently leaving a small void between each particle and the nanoscale cavity that contains it within the crystal. ${ }^{12}$ To understand the surface adsorption behavior of these anionic nanoparticles requires observing the nanoparticle-crystal interaction with a growth hillock "paused" at equilibrium. Such experiments demonstrated that the nanoparticles attach almost exclusively to step edges once they are bound to the calcite surface, implying highly specific adsorption and/or rapid surface diffusion to these features. ${ }^{12}$ Despite these useful mechanistic insights, the role of the chemistry of the anionic corona in mediating the 
nanoparticle-crystal surface interaction, as well as the incorporation effectiveness, remains poorly understood. Exploring these aspect of nanoparticle occlusion within calcite is the primary objective of the present study.

\section{Results}

Experimental Design. To effectively probe the role of surface chemistry in driving nanoparticle incorporation into calcite, we required a series of nanoparticles with tunable surface chemistry, ranging from strongly interacting to non-interacting. Polymerization-induced self-assembly $(\mathrm{PISA})^{29,30}$ via reversible addition-fragmentation chain transfer (RAFT) emulsion polymerization enables the convenient synthesis of sterically-stabilized diblock copolymer nanoparticles with identical hydrophobic cores but different surface chemistries conferred by the hydrophilic stabilizer chains. ${ }^{22,31}$ Moreover, the core-forming block can be selected to be optimal for the experiment. For example, an in situ AFM growth experiment works best with relatively hard, nonfilm-forming nanoparticles, which minimize tip effects and also nanoparticle deformation after adsorption. Similar nanoparticles have been previously utilized for the study of occlusion within calcite ${ }^{22,24,27,28,32}$ and zinc oxide ${ }^{33}$ : nanoparticles containing relatively long carboxylated stabilizer chains (e.g., poly(methacrylic acid) (PMAA)) that acquire strongly anionic character under the growth conditions $(\sim \mathrm{pH} 8)$ are most readily incorporated.

The specific diblock copolymer nanoparticles utilized in this study comprise a poly(benzyl methacrylate) (PBzMA) core-forming block and either a non-ionic poly(glycerol monomethacrylate) (Ph-PGMA), a carboxylic acid-tipped poly(glycerol monomethacrylate) (HOOC-PGMA) [i.e. a single anionic charge located at the chain terminus], or a highly anionic poly(methacrylic acid) (PMAA) steric stabilizer block (Scheme 1). The HOOC-PGMA particles were included in the series to provide particles with a negatively charged surface, but non-ionic 
corona. The single terminal anionic charge was conferred by selecting an appropriate carboxylic acid-functionalized RAFT chain transfer agent (CTA). We targeted a mean degree of polymerization of 100 for the hydrophobic core-forming PBzMA block to minimize the effects of nanoparticle size and deformation during calcite occlusion. As a result, these copolymer nanoparticles are relatively robust compared to the anionic nanoparticles used in previous AFM studies $^{12,27}$ and are subsequently less susceptible to AFM tip artifacts. For all of these diblock copolymer nanoparticles, however, there is in principle a micelle-unimer equilibrium, with a background concentration of individual copolymer chains (unimers). ${ }^{34}$ This concentration of molecularly-dissolved copolymer chains is finite but likely to be extremely low. To examine the effect of systematically increasing the anionic character of the stabilizer block, we grew calcite in the presence of each type of nanoparticle both in the bulk (ex situ) and also using in situ AFM. The combination of ex situ and in situ experiments allows us to make direct comparisons between the three types of nanoparticles at different length scales. 


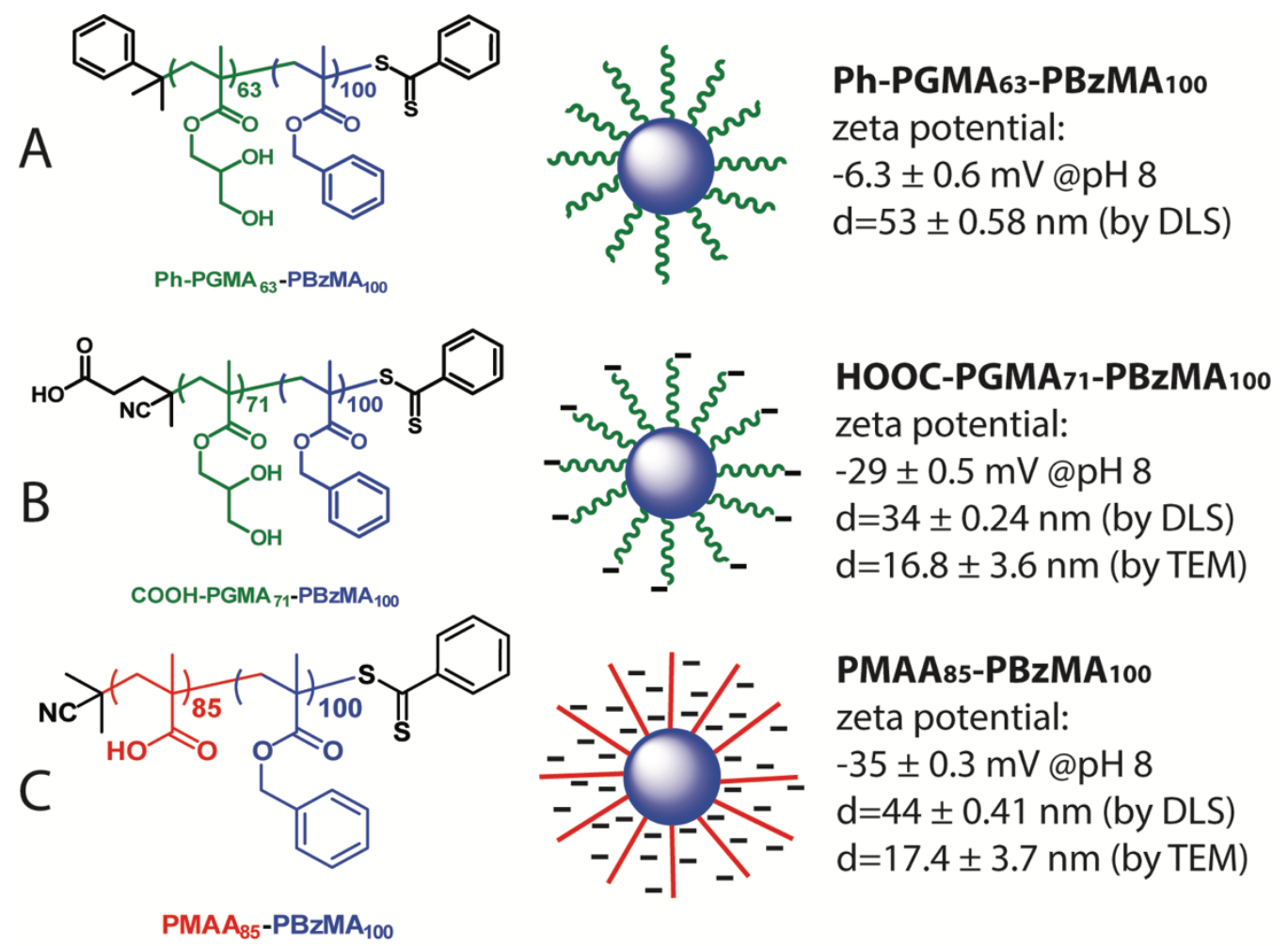

Scheme 1. Summary of the chemical structure and characterization of the three types of diblock copolymer nanoparticles used in this study: (A) non-ionic poly(glycerol monomethacrylate)stabilized poly(benzyl methacrylate) nanoparticles (Ph-PGMA $63-\mathrm{PBzMA}_{100}$ ); (B) weakly anionic poly(glycerol monomethacrylate)-stabilized poly(benzyl methacrylate) nanoparticles with a single carboxylic acid group located at the end of each PGMA stabilizer chain (HOOC-PGMA 71- $^{-}$ PBzMA $_{100}$ ); (C) strongly anionic poly(methacrylic acid)-stabilized poly(benzyl methacrylate) nanoparticles (PMAA $\left.85-\mathrm{PBzMA}_{100}\right)$.

Nanoparticle Characterization. Prior to any detailed AFM study of nanoparticle-calcite interactions, we characterized the nanoparticles themselves. First, the zeta potential and zaverage diameter were each determined as a function of $\mathrm{pH}$ (Figure 1). The zeta potential measured at $\mathrm{pH} 8$ (the $\mathrm{pH}$ used for calcite growth) for all three nanoparticle types is listed in 
Scheme 1. The non-ionic Ph-PGMA63-PBzMA 100 nanoparticles are slightly negative (-6.3 \pm 0.6 $\mathrm{mV})$, whereas both the HOOC-PGMA $71-\mathrm{PBzMA}_{100}$ and $\mathrm{PMAA}_{85}-\mathrm{PBzMA}_{100}$ nanoparticles are highly negatively charged $(-29 \pm 0.5 \mathrm{mV}$ and $-35 \pm 0.3 \mathrm{mV}$, respectively). Since zeta potential is sensitive to surface charge, rather than coronal charge, the similarity between these two particle types is not surprising. ${ }^{27,35-37}$ There is no $\mathrm{pH}$-dependence for the zeta potential of the non-ionic Ph-PGMA 63 -PBzMA 100 nanoparticles, whereas the HOOC-PGMA $71-\mathrm{PBzMA}_{100}$ and PMAA 85 PBzMA $_{100}$ nanoparticles acquire progressively greater anionic character at higher $\mathrm{pH}$ as the carboxylic acid groups become deprotonated. The diameter of the HOOC-PGMA $11-\mathrm{PBzMA}_{100}$ nanoparticles remains relatively constant at $\sim 34 \mathrm{~nm}$ while the $\mathrm{PMAA}_{85}-\mathrm{PBzMA}_{100}$ nanoparticles increase from $\sim 25 \mathrm{~nm}$ to $\sim 45 \mathrm{~nm}$ diameter between $\mathrm{pH} 4$ and $\mathrm{pH} 8$. This observation is consistent with polyelectrolyte brush behavior, where a higher $\mathrm{pH}$ causes swelling of the coronal layer via extension of the increasingly anionic chains ${ }^{38,39}$. Therefore, polyelectrolytic PMAA chains are likely to adopt a much more stretched conformation than the non-ionic PGMA chains due to coulombic repulsion between adjacent anionic groups, although crowding within the coronal layer means that the degree of ionization is reduced compared to free chains. The HOOC$\mathrm{PGMA}_{71}-\mathrm{PBzMA}_{100}$ nanoparticles do not possess polyelectrolytic stabilizer blocks and therefore do not exhibit such coronal swelling behavior. We used both transmission electron microscopy (TEM) and static AFM on poly(L-lysine)-treated mica in fluid to confirm that these PBzMAbased nanoparticles could be imaged after their adsorption at a planar surface (Fig. S1). Indeed, nanoparticle core diameters are very similar when imaged by TEM and AFM $(\sim 15 \mathrm{~nm})$. 


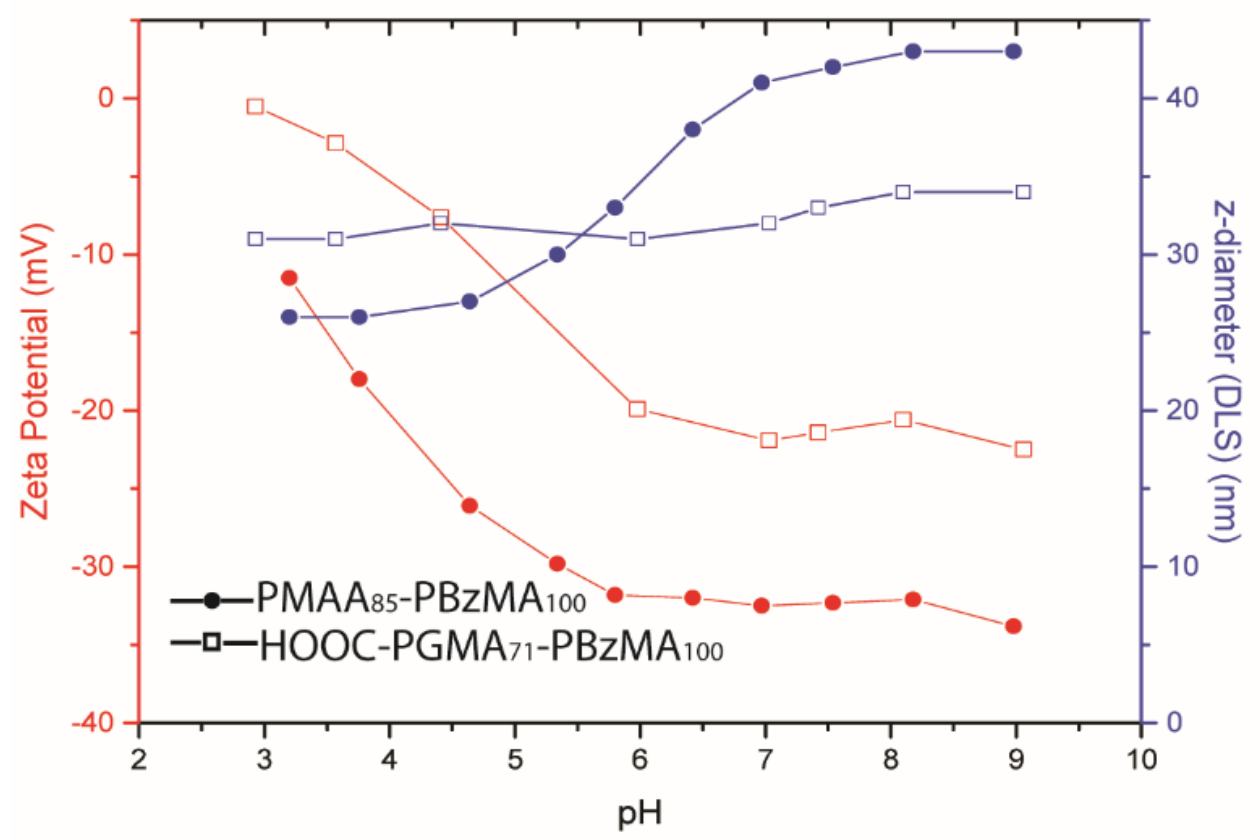

Figure 1. Variation of zeta potential (red) and DLS diameter (blue) with $\mathrm{pH}$ for both PMAA 85- $^{-}$ PBzMA $_{100}$ (filled circles) and HOOC-PGMA $11-$ PBzMA $_{100}$ (open squares) nanoparticles. Measurements were conducted in the presence of $1 \mathrm{mM} \mathrm{KCl}$ and titrated from basic to acidic $\mathrm{pH}$ using $\mathrm{HCl}$.

Calcite Growth Ex Situ. The most common method to examine the effect of additives, such as nanoparticles, on calcite formation is to add them to the aqueous solution in which the crystals are grown, as reported in various studies. ${ }^{4,22,24}$ In control experiments performed in the absence of any nanoparticles, rhombohedral crystals with smooth $\{104\}$ facets are formed (Fig. 2A). Crystals grown in the presence of non-ionic Ph-PGMA63-PBzMA 100 nanoparticles remain rhombohedral, as expected (Fig. 2B). However, if anionic nanoparticles are present during calcite formation, the crystal morphology is affected. Calcite rhombohedra grown in the presence of HOOC-PGMA 71 -PBzMA 100 nanoparticles have rounded edges and corners and much rougher $\{104\}$ facets, suggesting some weak interactions between these nanoparticles and growing crystal (Fig. 2C). The presence of PMAA $85-\mathrm{PBzMA}_{100}$ nanoparticles has a much more dramatic 
impact on the crystal morphology (Fig. 2D). The $\{104\}$ facets are retained as end-caps, but crystals become rounded and extended into bullet-like shapes, indicating a very strong interaction between such nanoparticles and the crystal lattice. Previous ex situ growth studies have shown that such a drastic change in morphology often correlates with a significant degree of incorporation ${ }^{4,19,40-42}$. Although these images provide a useful initial guide, direct observation at much smaller length scales using in situ AFM is required to characterize the true nature of the nanoparticle-crystal interaction.

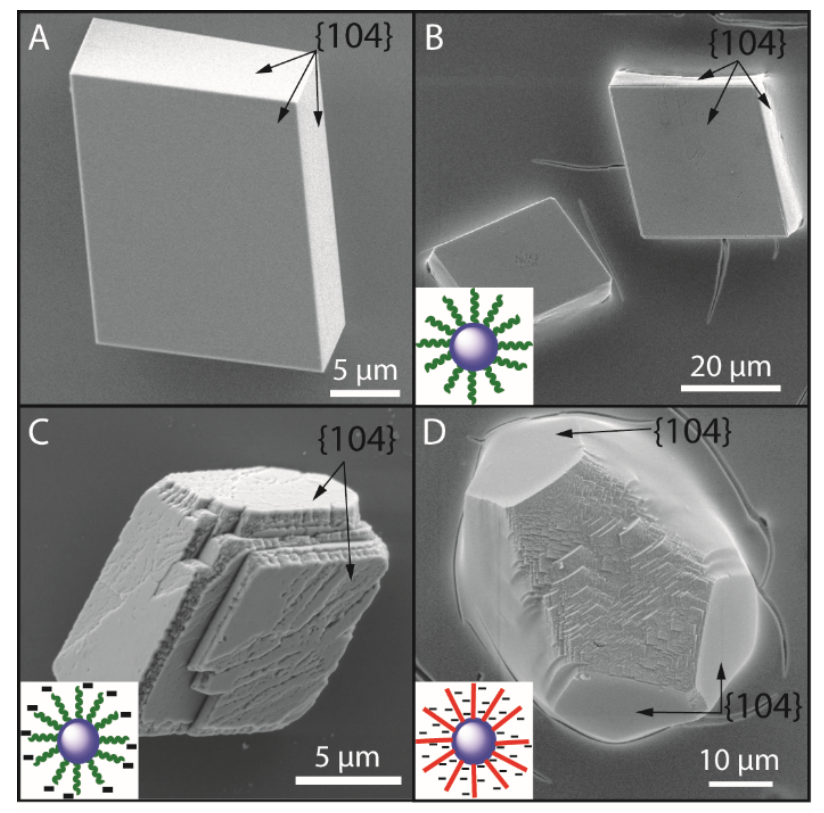

Figure 2. Representative scanning electron microscopy (SEM) images of calcite grown ex situ at $20{ }^{\circ} \mathrm{C}$ with the three types of nanoparticles used in this study. (A) Control calcite grown in the absence of any nanoparticles. (B) Representative crystals grown in the presence of non-ionic $\mathrm{Ph}$ PGMA $_{63}-\mathrm{PBzMA}_{100}$ nanoparticles. A cracked polymer film is evident in the background. (C) Representative crystal grown in the presence of weakly anionic HOOC-PGMA ${ }_{71}-\mathrm{PBzMA}_{100}$ nanoparticles. (D) Representative crystal grown in the presence of highly anionic $\mathrm{PMAA}_{85^{-}}$ PBzMA $_{100}$ nanoparticles. In each case the ammonia diffusion method was utilized at $\mathrm{pH} 8$ using $5 \mathrm{mM} \mathrm{CaCl}_{2}$ and a copolymer nanoparticle concentration of $0.75 \mathrm{wt} \%$. In all images, black arrows indicate calcite $\{104\}$ facets. 
In situ AFM. To conduct in situ AFM studies, growth solution is flowed over the surface of a freshly-cleaved single crystal of calcite using a fluid cell containing the AFM tip. ${ }^{43,44}$ Under carefully controlled conditions corresponding to relatively low supersaturation (supersaturation $\sigma$ is defined in Equation 1, where $A P$ is the activity product and $K_{s p}$ is the equilibrium solubility product), calcite grows via addition of $\mathrm{CaCO}_{3}$ units to hillocks, which are sources for atomic steps originating from screw dislocations within the crystal. ${ }^{43-45}$

$$
\sigma=\ln \left(\frac{A P}{K_{s p}}\right) \quad \text { Equation (1) }
$$

Observing in situ growth via this technique allows direct observation of nanoparticle-crystal interactions at conditions close to those found in Nature (ambient, room temperature, and a low degree of supersaturation). Based on previous AFM literature and classical crystal growth theories, we anticipated three likely modes of interaction for nanoparticles with a growing calcite crystal. 7,11,45 These are: (i) initial attachment followed by detachment from the surface, (ii) attachment with subsequent incorporation into the crystal, as previously described, ${ }^{12}$ and (iii) an intermediate scenario whereby a nanoparticle "hovers" on the calcite surface, while steps continue to flow beneath it. ${ }^{11}$ In principle, all three interactions could occur within a population of nanoparticles at the calcite surface. The specific nanoparticle surface chemistry is expected to determine the relative contributions of these three modes of interaction for a given nanoparticle type. With sufficient data, we can analyze the behavior of an ensemble of nanoparticles to characterize their behavior at the surface for each class of nanoparticles considered here (i.e., non-ionic, weakly anionic, or strongly anionic). 


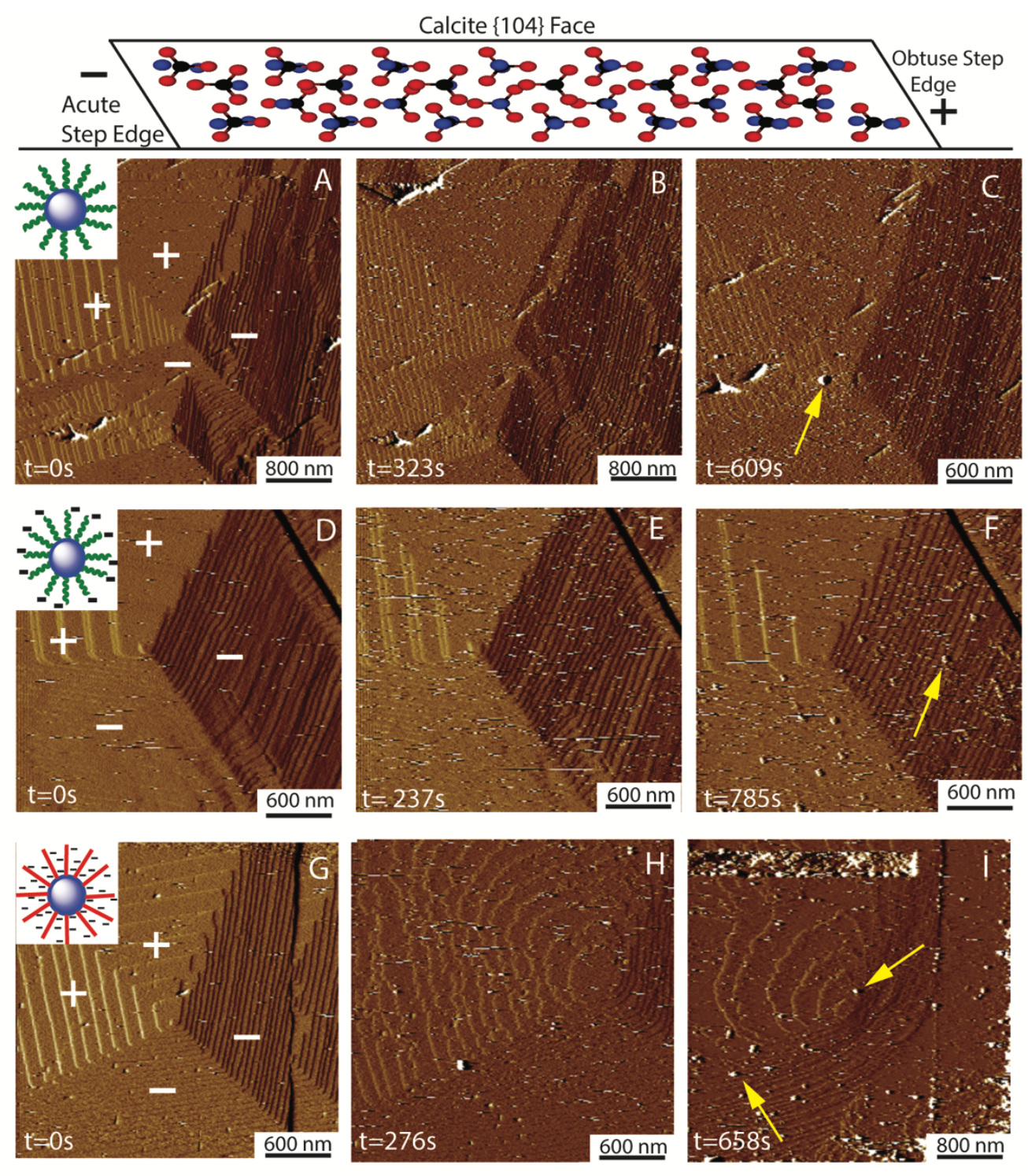

Figure 3. Time-resolved in situ AFM studies of calcite growth in the presence of three types of sterically-stabilized diblock copolymer nanoparticles $(\sigma=1.15$, nanoparticle concentration $=$ $0.00075 \mathrm{wt} \%)$. Zero time $(\mathrm{t}=0)$ refers to when the nanoparticle-containing growth solution is first added to the AFM cell. Top schematic shows a cross-section through a calcite growth hillock showing the side view of obtuse $(+)$ and acute (-) step edges (blue - calcium, red oxygen, black - carbon). A-C: growth in the presence of non-ionic Ph-PGMA $63-\mathrm{PBzMA}_{100}$ nanoparticles. D-F: growth in the presence of weakly anionic HOOC-PGMA $71-\mathrm{PBzMA}_{100}$ nanoparticles. G-I: growth in the presence of strongly anionic PMAA $85-P_{8} \mathrm{PAA}_{100}$ nanoparticles. Yellow arrows indicate particles that have attached to the crystal surface. For each particle type, 
images are tip deflection (color scale $\pm 20 \mathrm{mV}$ ) taken in contact mode. The (+) and (-) indicate the obtuse and acute steps of the growing calcite hillock, respectively. Movies S1-S3 depict a more complete series of images.

Calcite growth studies using in situ AFM allows direct observation of the nanoparticlecrystal interactions (Fig. 3 and Movies S1-S3). A control growth hillock forms in the absence of any nanoparticles (Figs. 3A, D, G). Once such a hillock is identified, the growth solution is switched to one that contains nanoparticles and is of identical ionic composition, ionic strength, and $\mathrm{pH}$. Then the nanoparticle-crystal interactions can be directly imaged under growth conditions. The Ph-PGMA $63-\mathrm{PBzMA}_{100}$ nanoparticles (Figs. 3A-C; S2) have negligible effect on the growing hillock and minimally interact with the calcite surface, although they are most likely present very close to the calcite-solution interface (note the increased noise in Figs. 3B,C). Such observations are fully consistent with the unmodified calcite crystals shown in Figure 2B. The HOOC-PGMA $71-\mathrm{PBzMA}_{100}$ nanoparticles bind to the acute steps of the growing hillock (Figs. 3E, F) but do not modify the step edges, which is similar to the minimal changes in morphology observed for the calcite crystal in Figure 2C. In striking contrast, the strongly anionic PMAA ${ }_{85-}$ PBzMA $_{100}$ nanoparticles initially cause roughening of both the obtuse and acute steps, leading to hillock rounding (Fig. 3H). This hillock modification is consistent with the bullet-like morphology of the calcite crystal in Fig. 2D. Subsequently, the hillock becomes heavily decorated by nanoparticles adsorbed on the acute steps (Fig. 3I). 

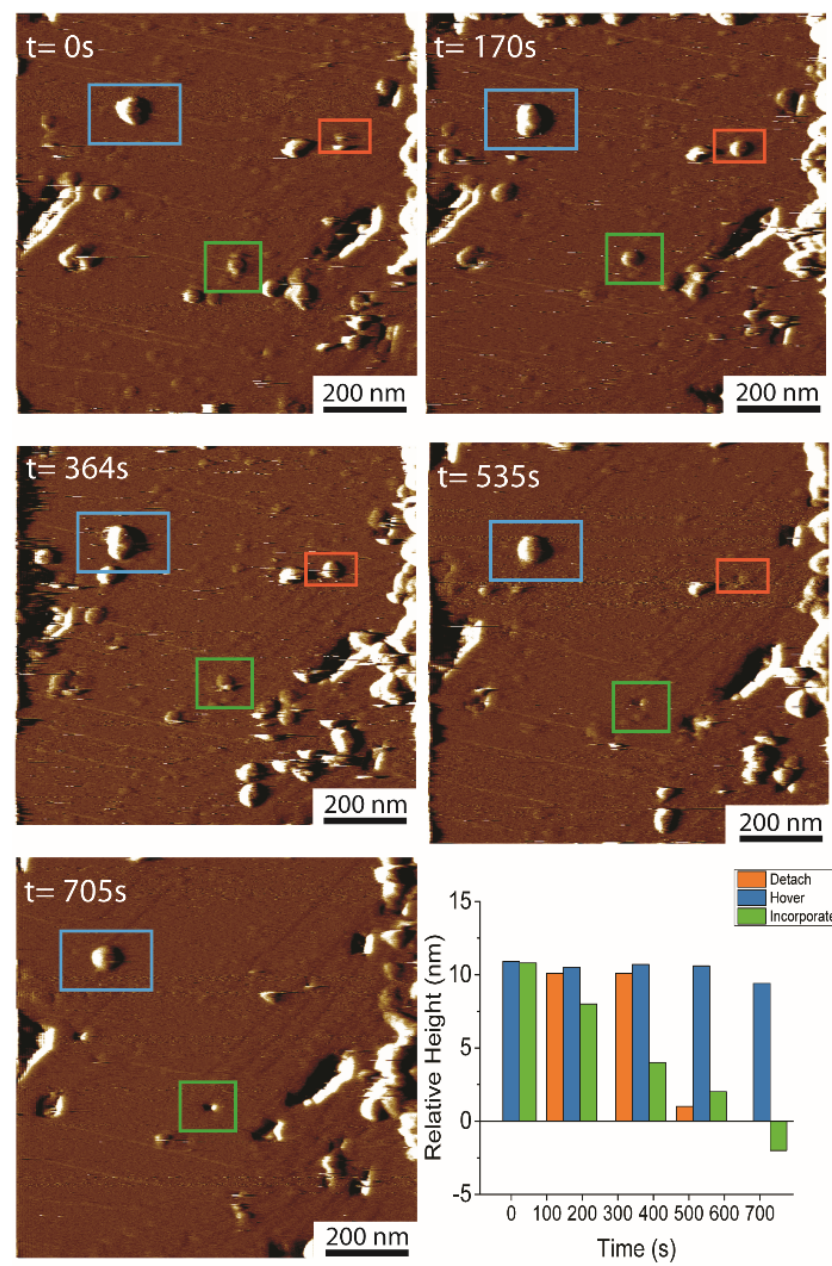

Figure 4. High resolution in situ AFM images of the highly anionic $\mathrm{PMAA}_{85}-\mathrm{PBzMA}_{100}$ nanoparticles interacting with a growing calcite face at $\sigma=1.15$. The height measurements in the bottom right graph are from line scan measurements of the highlighted nanoparticles. Over time, three types of behavior can be observed, although the highlighted nanoparticles start at similar relative heights. The orange nanoparticle attaches between the first and second image, then detaches between the third and fourth image. There is a gradual reduction in the height of the green nanoparticle until it is apparently incorporated within the growing crystal. The blue nanoparticle "hovers" on the surface, staying attached but not becoming incorporated within the crystal. This Figure is also available as Movie S4. Comparable behavior is highlighted with line scans for each particle type in SI Figures S3 and S4. 
Nanoparticle-Crystal Interactions. Imaging the nanoparticle-crystal interactions at higher resolution reveals three types of behavior, (Figs. 4, S3, S4): (i) after initial attachment, the nanoparticle detaches from the surface (orange box); (ii) the nanoparticle attaches to the surface and there is a progressive reduction in its height as it becomes incorporated within the crystal (green box), producing a small cavity similar to those reported by De Yoreo and co-workers. ${ }^{12}$; (iii) the nanoparticle attaches to the calcite surface and "hovers" above it without changing its height significantly until either it becomes detached or is eventually occluded (blue box). Close inspection of the image series shown in Fig. 4 confirms multiple examples of these three modes of interaction.

Sampling larger populations of nanoparticles is required to quantify the relative contribution made by each interaction mode for each of the three classes of nanoparticles. Unfortunately, there were too few of the non-ionic (uncharged) Ph-PGMA-PBzMA nanoparticles interacting with the surface to obtain any statistically significant distribution for this nanoparticle type, so we focused on a close comparison of the behavior of the HOOCPGMA $_{71}-\mathrm{PBzMA}_{100}$ and PMAA $85-\mathrm{PBzMA}_{100}$ nanoparticles. By tracking individual nanoparticle heights over time, we can gain a better understanding of their behavior and hence reveal the relative contributions made by each of the three modes of interactions (Figure 5 and Supplementary Figures S5-S7). Nanoparticles were selected for inclusion in statistics only if (i) their initial height was between 9 and $20 \mathrm{~nm}$ (so as to minimize the unwanted inclusion of any nanoparticle aggregates) and (ii) there were no other nanoparticles within $50 \mathrm{~nm}$ of the measured particle (see Fig. S5). Qualitatively, more PMAA $85-\mathrm{PBzMA}_{100}$ than HOOC-PGMA71-PBzMA 100 nanoparticles bind to the calcite surface within an equivalent amount of time. Tracking nanoparticle heights as a function of time reveals that the strongly anionic $\mathrm{PMAA}_{85}-\mathrm{PBzMA}_{100}$ 
and weakly anionic HOOC-PGMA $71-\mathrm{PBzMA}_{100}$ nanoparticles appear to become incorporated within calcite at approximately the same rate, implying a similar incorporation mechanism for both types of nanoparticles (Figs. 5B and S6). A summary of the three modes of interactions reveals that a similar fraction of both types of nanoparticles are incorporated $(\sim 50 \%$ of the population), whereas the fraction of particles that hover and detach vary significantly between the two types. For the PMAA $85-\mathrm{PBzMA}_{100}$ nanoparticles, $\sim 35 \%$ hover while only $\sim 15 \%$ become detached. Conversely, $\sim 15 \%$ of the HOOC-PGMA $71-$ PBzMA $_{100}$ nanoparticles hover while $\sim 30 \%$ detach (Fig. 5A). When the nanoparticles are sorted with respect to which type of interaction they undergo as a function of time, it becomes evident that most of the PMAA ${ }_{85}-$ $\mathrm{PBzMA}_{100}$ nanoparticles either begin incorporating into the calcite lattice or detach (Fig. S7). Furthermore, it appears that nanoparticles that bind to the surface at later times are much more likely to "hover". 

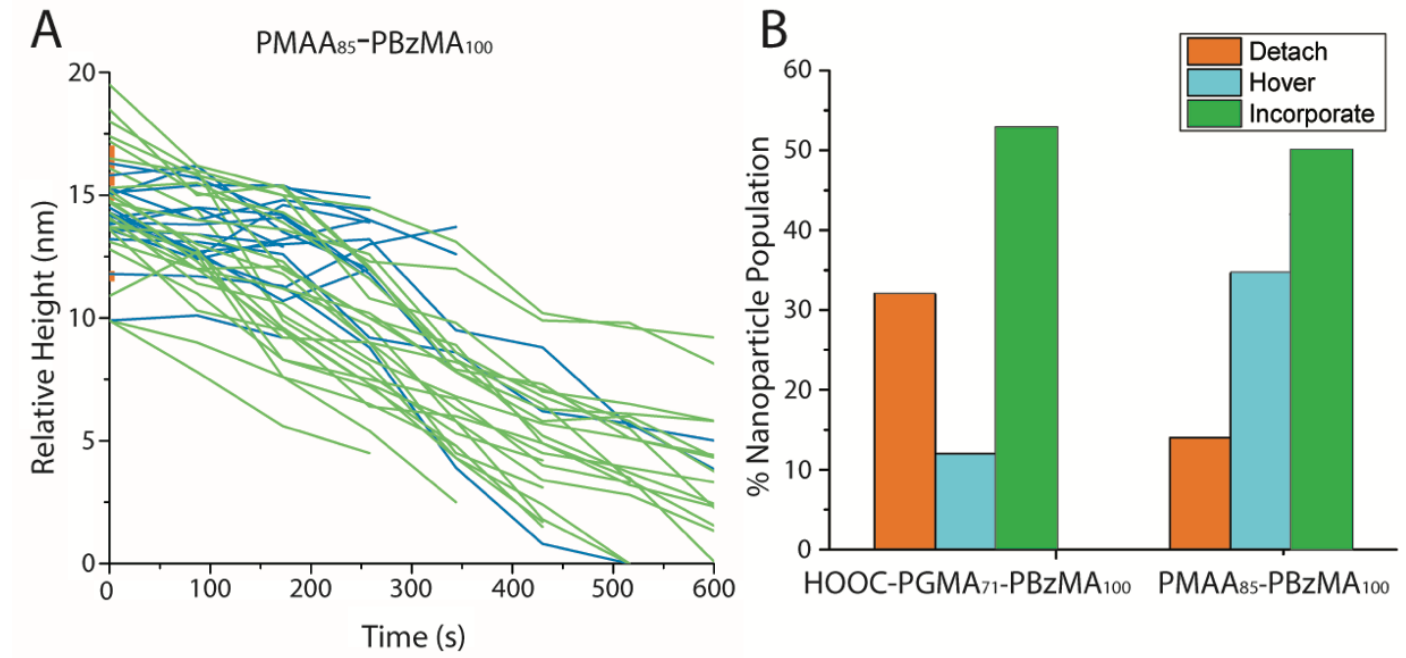

Figure 5. Comparison of the three modes of interaction of HOOC-PGMA71-PBzMA 100 and PMAA $_{85}-\mathrm{PBzMA}_{100}$ nanoparticles with calcite as determined by in situ AFM studies. (A) A plot of height vs. time data for individual $\mathrm{PMAA}_{85}-\mathrm{PBzMA}_{100}$ nanoparticles. Each relative height trace corresponds to an individual nanoparticle tracked across multiple AFM images and begins at the first image at which a particle is measured. Three types of nanoparticle behavior were observed: (i) detachment from the surface within one image (orange lines; $n=7$ ), (ii) hovering on the surface before either detachment or incorporation (blue lines; $n=17$ ), and (iii) incorporation directly into the crystal (green lines, $n=26$ ). Incorporation appears to occur at similar rates for all nanoparticles. Qualification for the designation of "hovering" required less than $10 \%$ variation in the observed nanoparticle height for at least three consecutive images $(\sim 5$ minutes real time). A comparable set of line traces obtained for HOOC-PGMA $71-\mathrm{PBzMA}_{100}$ nanoparticles is shown in Fig. S6. (B) Histogram showing the relative proportion of each nanoparticle population to exhibit detachment, hovering, or incorporation within the growing calcite crystal. ( $n=50$ for both particle types accumulated over 4 repeated, equivalent experiments with HOOC-PGMA $71-\mathrm{PBzMA}_{100}$ and 3 repeated experiments with PMAA85$\mathrm{PBzMA}_{100}$ ). See Methods section for further details on image analysis and nanoparticle selection. 


\section{Discussion}

The chemistry of the steric stabilizer chains (and resulting anionic charge density of the nanoparticles) clearly dictate the distribution of the mode of interaction of the nanoparticles at the crystal surface. The non-ionic Ph-PGMA $63-\mathrm{PBzMA}_{100}$ nanoparticles have essentially no interaction with growing calcite. The weakly anionic HOOC-PGMA $71-\mathrm{PBzMA}_{100}$ nanoparticles only slightly modify the morphology of the calcite crystals but still interact strongly enough for any given nanoparticle to potentially participate in one of the three modes of interaction. The strongly anionic $\mathrm{PMAA}_{85}-\mathrm{PBzMA}_{100}$ nanoparticles attach in much greater numbers to the calcite surface and exhibit each of the three modes of interaction. The in situ AFM data summarized in Figure 5 lead to the following three important questions regarding the nature of the nanoparticlecrystal interaction. (1) How does the variation in coronal charge density affect initial nanoparticle binding to the surface? (2) Why do only the PMAA $85-\mathrm{PBzMA}_{100}$ nanoparticles "hover" in large numbers? (3) Why do nanoparticles that bind later tend to "hover" rather than become incorporated directly?

Charge Corona Effect on Particles in Solution. We cannot properly analyze nanoparticle-crystal interactions without first considering the chemical structure of the nanoparticles in the growth solution. Both Ph-PGMA63-PBzMA 100 and HOOC-PGMA71-PBzMA 100 possess flexible stabilizer chains with either zero or relatively low charge density. In contrast, the PMAA 85 stabilizer chains should act as a polyelectrolyte brush because of its highly anionic nature at $\mathrm{pH}$ 8. ${ }^{38,39}$ When immersed in a solution at a $\mathrm{pH}$ above its $p \mathrm{~K}_{\mathrm{a}}$, a PMAA brush becomes swollen and extended due to deprotonation, resulting in coulombic repulsion between the highly anionic chains. ${ }^{38}$ In Figure 1, DLS studies indicate that the mean diameter of the PMAA $85-\mathrm{PBzMA}_{100}$ nanoparticles substantially increases on switching from below $\mathrm{pH} 4(25 \mathrm{~nm})$ to $\mathrm{pH} 8(45 \mathrm{~nm})$. 
Similar pH-dependent behavior has been observed for PMAA brushes ${ }^{38}$ and typically occurs at a much higher $\mathrm{pH}(\mathrm{pH} 9)$ when compared to the $p \mathrm{~K}_{\mathrm{a}}$ of 5.5 for free PMAA. However, the behavior of these nanoparticles, with similar grafting densities to a dense brush, but with an interface that is curved on the length scale of the chains, is intermediate between that of isolated PMAA chains and dense planar PMAA brushes and the apparent $p \mathrm{~K}_{\mathrm{a}}$ is only shifted by at most 1 $\mathrm{pH}$ unit. Both polyelectrolyte brushes and free chains are known to be strongly affected by ionic strength; in particular, the brushes can become swollen with increasing salt concentration. ${ }^{38,39}$ Such swelling is an osmotic effect driven by cation binding to the PMAA chains, since this interaction generates an osmotic pressure via a net increase in charge density. ${ }^{38}$ The PMAA $_{85}$ stabilizer chains, however, eventually become saturated with ions and collapse (the so-called "salted brush" regime). ${ }^{38}$ Therefore, PMAA stabilizer chains should become highly extended in calcite growth solution $\left(1.8 \mathrm{mM} \mathrm{Ca}^{2+}\right.$ and $36 \mathrm{mM} \mathrm{Na}^{+}$ions at $\left.\mathrm{pH} 8\right)$ as the ionic strength is in the swollen regime and far from the "salted out" collapse. In contrast, the HOOC-PGMA 71 stabilizer chains are not polyelectrolytes and thus do not exhibit such swelling behavior.

A Model for Nanoparticles Interacting with a Growing Crystal. The observations made in these in situ AFM experiments combined with previous studies of peptides on calcium carbonate and calcium oxalate lead us to present a model that describes the likely interactions of anionic diblock copolymer nanoparticles with growing calcite crystals in the low supersaturation regime. This model is depicted in Scheme 2: the nanoparticle first binds to the surface via electrostatic interactions between the anionic carboxylate groups and the cationic $\mathrm{Ca}^{2+}$ ions in the crystal lattice. On attachment, relatively fast nanoparticle diffusion occurs to the step edges in most cases. However, if the particles happen to attach at a step edge, they may not diffuse at all. De Yoreo and co-workers clearly demonstrated that nanoparticles are bound almost exclusively to 
the step edges under equilibrium conditions, thus suggesting relatively fast surface diffusion, at least initially. ${ }^{12}$ Once a nanoparticle becomes strongly bound to the surface, its diffusion becomes hindered by the ionic bond(s) between the charged group(s) and the surface. Depending on the local solution chemistry, the bound nanoparticle can either be in a swollen or a collapsed state. In the swollen state, the anionic stabilizer chains are extended, so the $\mathrm{Ca}^{2+}$ and $\mathrm{CO}_{3}{ }^{2-}$ ions can still access the step edge; this binding configuration allows the step to propagate under the nanoparticle, which "hovers" on the surface. If the PMAA 85 chains are instead "salted out", then the nanoparticle collapses onto the surface and access to the step edge is blocked. Thus the step is forced to grow around the nanoparticle, leading eventually to nanoparticle incorporation within the crystal. Swollen nanoparticles can also subsequently collapse, hence "hovering" nanoparticles can also eventually become occluded. In a related study of peptides interacting with calcium oxalate crystals, a similar multistep time-dependent adsorption model is proposed. ${ }^{16}$ 


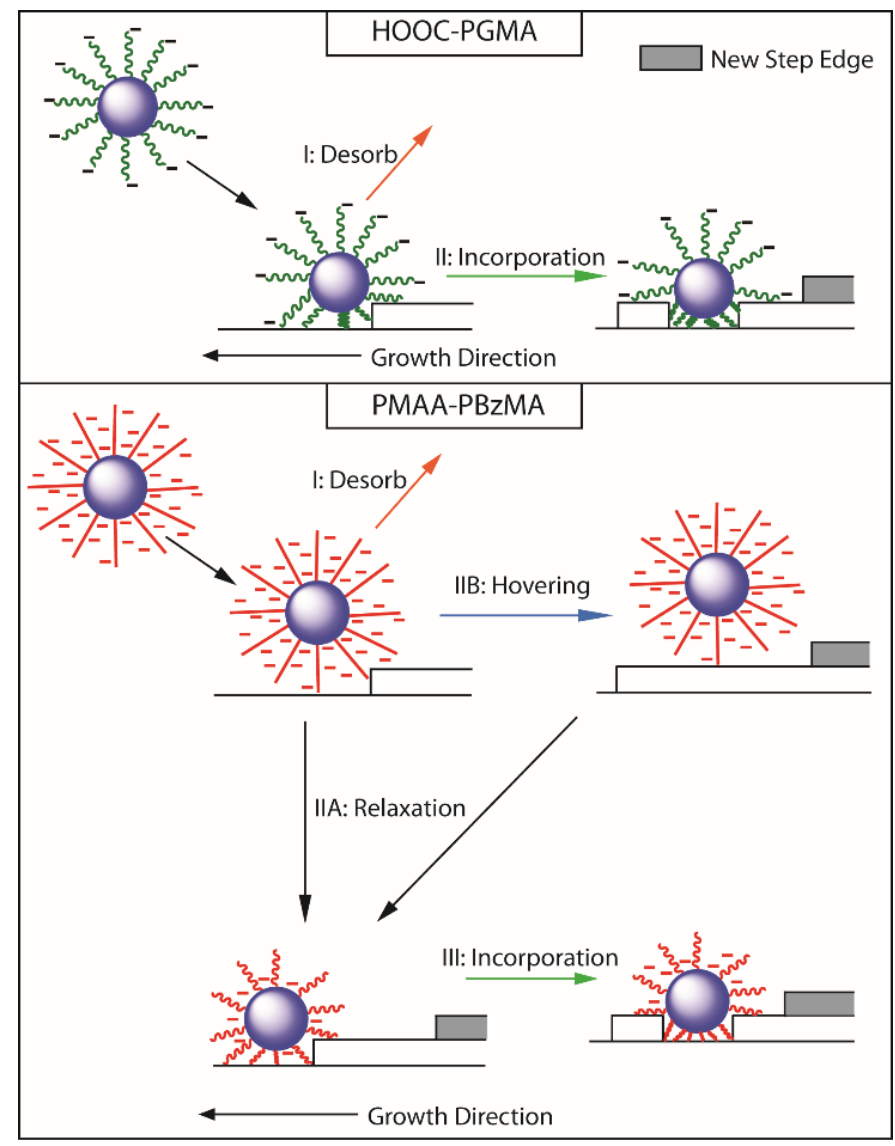

Scheme 2. Schematic representation of the three possible modes of interaction of two types of anionic nanoparticles interacting with a growing calcite surface. On contact with the surface, the nanoparticles first undergo fast diffusion to become bound at a step edge. Subsequent relaxation of the copolymer chains determines to what extent the growth species $\left(\mathrm{Ca}^{2+}\right.$ and $\mathrm{CO}_{3}{ }^{2-}$ ions $)$ can access binding sites on the step edge. Hence the nanoparticle either (I) detaches or (II) continues to interact with the surface by "hovering". For the highly anionic PMAA $85-\mathrm{PBzMA}_{100}$ nanoparticles, the copolymer chains can either (IIB) remain rigid (slow relaxation) to allow access of growth ions to the step edge or (IIA) relax quickly, thus blocking growth species. For the HOOC-PGMA 71 stabilizer chains, faster relaxation (II) blocks ions from diffusing to the step edge. When such mass transport is blocked, the collapsed nanoparticle becomes occluded as the step grows around it.

Once the nanoparticles interact with the calcite surface, Figure 5B illustrates the strikingly different behavior observed for the two types of anionic nanoparticles, which both 
have similar zeta potentials at $\mathrm{pH}$ 8. For the HOOC-PGMA ${ }_{71}-\mathrm{PBzMA}_{100}$ nanoparticles, there is only a single terminal anionic carboxylate group per chain, so binding to the calcite surface is relatively weak and the nanoparticles tend to either detach or become incorporated. In contrast, the $\mathrm{PMAA}_{85}-\mathrm{PBzMA}_{100}$ nanoparticles can bind more strongly and access a much greater number of binding states as a result of the cooperativity conferred by the highly anionic PMAA 85 chains. Therefore, for any given $\mathrm{PMAA}_{85}-\mathrm{PBzMA}_{100}$ nanoparticle attachment, subsequent "hovering" or incorporation within the growing crystal lattice is much more favorable as compared to the HOOC-PGMA ${ }_{71}-$ PBzMA $_{100}$ nanoparticles ( $86 \%$ vs. $68 \%$ of all particles hover or incorporate). Our in situ data are consistent with earlier (albeit more qualitative) ex situ data; ${ }^{22,23,28}$ the zeta potential of the nanoparticles alone is not sufficient to predict efficient occlusion. Instead, the precise nature of the nanoparticles and their surface charge density must be considered.

The nature of the stabilizer chains accounts for the observed interactions. When a nanoparticle initially attaches to the surface, it either remains adsorbed or subsequently becomes detached. If it stays bound, the long stabilizer chains undergo relaxation to maximize contact with the surface. For the HOOC-PGMA ${ }_{71}-\mathrm{PBzMA}_{100}$ nanoparticles, such relaxation is likely to be fast, since the stabilizer chains only possess a single terminal anionic charge and the chains never adopt a stretched conformation. The $\mathrm{PMAA}_{85}-\mathrm{PBzMA}_{100}$ nanoparticles most likely have more extended stabilizer chains so relaxation is more complex, possibly involving both fast and slow relaxation modes. For highly swollen $\mathrm{PMAA}_{85}-\mathrm{PBzMA}_{100}$ nanoparticles with extended stabilizer chains, relaxation is likely to be relatively slow. Such stretched chains are likely to be highly permeable to ions, making it easier for the crystal to grow beneath attached nanoparticles. "Hovering" occurs when such nanoparticles remain at the calcite surface in their swollen state for some time prior to ion-induced collapse. $\mathrm{PMAA}_{85}-\mathrm{PBzMA}_{100}$ nanoparticles are more likely to 
display this hovering behavior because they are more prone to undergo slower corona relaxation. For either type of nanoparticle, once the stabilizer chains relax at the surface, the step edge is forced to grow around the nanoparticle, leading to its incorporation within the crystal lattice. Effect of free polymer in solution. Upon adding the strongly anionic $\mathrm{PMAA}_{85}-\mathrm{PBzMA}_{100}$ nanoparticles to the growing calcite crystal, in situ AFM studies reveal a substantial change in the hillock morphology (including roughening of the step edges) before these nanoparticles are observed at the surface (Fig. 3H). Similar local roughening is common for small molecules interacting with calcite, so the roughening that we observe may indicate the presence of soluble PMAA chains (a likely impurity in the PISA synthesis) or individual copolymer chains (from a micelle-unimer equilibrium). In either case, the smaller size of free PMAA 85 homopolymer or molecularly-dissolved copolymer should lead to much faster diffusion to the growing crystal surface compared to the nanoparticles. Any free anionic (co)polymer would bind tightly to the step edges, which could cause a change in hillock morphology even if this soluble component were present in a relatively low concentration ${ }^{4}$. The step roughening may enhance initial nanoparticle attachment to the surface by generating more kink sites (high energy binding sites) on the step edges ${ }^{45}$. The weakly anionic HOOC-PGMA $71-\mathrm{PBzMA}_{100}$ and non-ionic PhPGMA $_{63}-\mathrm{PBzMA}_{100}$ nanoparticles behave very differently from the strongly anionic $\mathrm{PMAA}_{85}$ PBzMA $_{100}$ nanoparticles. There is little or no hillock modification over a comparable time scale (Fig. 3B. 3E), and step progression is clearly observed with no change in morphology (Fig. S3).

A surprising feature of the histograms shown in Figure S7 is that most of the nanoparticles that bind later remain in their swollen state and "hover." Though they may subsequently become incorporated, the concept of a time-dependent distribution of nanoparticles between incorporation and "hovering" is surprising because it is usually assumed that a steady 
state has been attained in such experiments, not least because the inflowing solution supplies a constant concentration of nanoparticles and growth units (i.e., $\mathrm{Ca}^{2+}$ and $\mathrm{CO}_{3}{ }^{2-}$ ions) for the crystal. However, this assumption ignores the potential influence of free (co)polymer chains in solution. The gradual build-up of adsorbed (co)polymer could provide an additional barrier to nanoparticles adopting their collapsed state at the surface. This process would increase the relative fraction of particles in the hovering state, thus explaining the apparent time dependence observed for both types of nanoparticles. In principle, an increase in "hovering" over time could also be induced by the AFM tip interacting with nanoparticles under continuous imaging (see Supporting Information for further discussion of possible tip interactions). In view of this possible imaging artifact, we ceased imaging for a few minutes between each series of images prior to resuming AFM studies. This precaution had no discernible effect on our observations.

\section{Conclusions}

Previous work has shown that atoms must be comparable in size to $\mathrm{Ca}^{2+}$ to substitute into the calcite lattice, while small molecules require negatively-charged functional groups and nanoparticles must have highly anionic coronas in order to become incorporated within calcite. In the latter case, the number of anionic carboxylate groups per chain is a key factor in governing the nanoparticle-crystal interaction. The in situ AFM studies detailed in this report provide important new insights regarding the roles of both surface chemistry and charge density in determining the precise mode of interaction between the nanoparticles and crystal surface. Specifically, we provide the first detailed quantification of the distribution of detachment, "hovering", and incorporation behaviors as a function of surface chemistry. For highly anionic nanoparticles, we suggest that the conformational transition from extended to collapsed steric stabilizer chains is a primary factor in determining the surface interaction, such that biasing the 
system towards collapsed chains enables incorporation to proceed. This study has provided new insights into nanoparticle-crystal interactions in real time on the nm length scale; our observations should inform the design of next-generation organic nanoparticles that enable the extent of occlusion within host crystals to be maximized. For example, tuning solution chemistry to promote collapse of the polyelectrolyte corona could be used to control the amount of nanoparticle incorporation. Moreover, appropriate consideration of how nanoparticles interact with the surface hydration layer of calcite could also help to optimize the extent of occlusion. ${ }^{46,47}$ Furthermore, our model for nanoparticle-crystal interactions is likely to be applicable to other host crystals, ultimately leading to the rational design of single crystal composites that pair disparate materials within a single structure.

\section{Materials and Methods}

Particle Synthesis. Synthesis of Ph-PGMA, HOOC-PGMA and PMAA macro-CTAs via RAFT solution polymerization has been described in detail elsewhere. ${ }^{24,27}$ The synthesis of stericallystabilized diblock copolymer nanoparticles via RAFT emulsion polymerization of BzMA was recently reported by Armes and co-workers ${ }^{48}$.

Dynamic light scattering and Zeta Potential. Dynamic light scattering (DLS) and zeta potential measurements were performed at $25^{\circ} \mathrm{C}$ using a Zetasizer Nano-ZS instrument (Malvern Instruments, UK) at a fixed scattering angle of $173^{\circ}$ using Dispersion Technology Software version 6.20. Copolymer dispersions were diluted in $1 \mathrm{mM} \mathrm{KCl}$. Aqueous dispersions were adjusted to $\mathrm{pH} 9$ using $\mathrm{KOH}$ and titrated to approximately $\mathrm{pH} 3$ with $\mathrm{HCl}$.

Transmission electron microscopy (TEM). TEM studies were conducted using a Philips CM 100 instrument operating at $100 \mathrm{kV}$ and equipped with a Gatan $1 \mathrm{k}$ CCD camera. Diluted 
nanoparticle dispersions $(\sim 0.10 \% \mathrm{w} / \mathrm{w})$ were dried on freshly glow-discharged carbon-coated copper/palladium grids (Agar Scientific, UK) and subsequently exposed to $0.75 \mathrm{w} / \mathrm{v} \%$ uranyl formate solution for $30 \mathrm{~s}$ at $20^{\circ} \mathrm{C}$ prior to analysis.

Ex Situ Calcite Growth. Crystals were grown in a desiccator via the classical ammonia diffusion method $^{49}$. All samples were $10 \mathrm{~mL}$ in total volume and used $5 \mathrm{mM} \mathrm{CaCl}_{2}$ solution in Petri dishes. Crystals were grown on glass cover slips, which were sonicated for 5 min each in DI water $(18.6 \mathrm{M} \Omega)$ and ethanol to remove any surface contamination before being placed in the growth solution. The nanoparticle dispersions (15 wt\%) were used neat in $0.5 \mathrm{~mL}$ aliquots for each sample. Crystals were allowed to grow in the desiccator saturated with ammonium carbonate vapor for two days. Then the glass slides containing the calcite crystals were removed and dried using $\mathrm{N}_{2}$ gas. The slides were then mounted on aluminum SEM imaging stubs and coated with $\sim 100 \mathrm{~nm}$ of conductive carbon before being imaged using a Tescan MIRA3 FESEM.

In Situ AFM Experiments. Imaging experiments were performed using a Multimode AFM with a Nanoscope III Controller (Digital Instruments) using the method reported by De Yoreo and coworkers. ${ }^{12}$ Solutions were prepared by adding $5 \mu \mathrm{L}$ of $15 \mathrm{wt} \%$ nanoparticle dispersion (final copolymer concentration $=5 \mu \mathrm{M})$ to premade aqueous solutions of $\mathrm{CaCl}_{2}(1.8 \mathrm{mM})$ and $\mathrm{NaCl}$ (39.4 mM). For imaging, geologic calcite (Iceland Spar, Ward's Scientific) was glued with epoxy to the steel AFM sample disk with a freshly-cleaved $\{104\}$ face for imaging. Cleaving of the calcite crystals was performed using a precision chisel and razor blade. Using a commercial fluid cell (MTFML, Bruker Nano) and a syringe pump to control fluid flow, growth solution was flowed through the cell at $20^{\circ} \mathrm{C}$ at a typical rate of $0.30 \mathrm{~mL} \mathrm{~min}^{-1}$. Imaging was performed using Bruker Nano DNP-S model cantilevers with a nominal radius of curvature for the tip of less than 
$10 \mathrm{~nm}$. Images of the calcite surface were recorded in either contact mode (primarily) or tapping mode using scan rates of $\sim 3 \mathrm{~Hz}$ and typically 256 lines per image. The key to imaging the nanoparticles is to minimize the force applied by the tip, otherwise unacceptable levels of nanoparticle displacement were observed. Even in the best case scenario, there was clear (but consistent) movement of the nanoparticles by the tip towards the edges of the image. However, in the minimal force case, this tip effect was consistent and reproducible (see Supplementary Information and Fig. S8). Detailed imaging areas were typically $1 \mathrm{x} 1,3 \times 3$, or $5 \times 5 \mu \mathrm{m}$. For consistent image sequences, images were always recorded in the up direction. For imaging the anionic nanoparticles adsorbed onto mica, the mica was freshly cleaved (using a razor blade and tape) and treated with $0.15 \%$ poly(L-Lysine) for $5 \mathrm{~min}$ and gently rinsed with DI water for $1 \mathrm{~min}$ to ensure its surface charge reversal and hence promote nanoparticle binding to the surface.

AFM Image Analysis. Images were analyzed using Nanoscope Analysis software. Heights were determined through individual line scans across at least six images (or until the nanoparticle either detached from the surface or became fully incorporated). All nanoparticles were tracked by hand to ensure there was no double counting. Mean surface residence times were determined by recording the time at the last image before the nanoparticle changed significantly in height. For the $\mathrm{PMAA}_{85}-\mathrm{PBzMA}_{100}$ nanoparticles, three different experiments were conducted to analyze 50 nanoparticles. For the HOOC-PGMA $71-P_{32 M A} 100$ nanoparticles, four separate experiments yielded a total of 50 nanoparticles for analysis. During analysis comparisons among experiments were made to check that data from each experiment showed similar distributions of behaviors.

Supporting Information: Characterization of polymeric nanoparticles, AFM images during calcite growth with $\mathrm{Ph}_{-} \mathrm{PGMA}_{63}-\mathrm{PBzMA}_{100}$ nanoparticles, representative AFM line scans of 
nanoparticles, method used to track nanoparticles, nanoparticle tracking for HOOC-PGMA71-

$\mathrm{PBzMA}_{100}$ nanoparticles, nanoparticle tracking for $\mathrm{PMAA}_{85}-\mathrm{PBzMA}_{100}$ nanoparticles, histogram for HOOC-PGMA ${ }_{71}-\mathrm{PBzMA}_{100}$ nanoparticle behavior with time, AFM tip effects, movies corresponding to Figs. 3A-C, 3D-F, 3G-I, 4.

\section{Acknowledgements}

This work was funded by a joint National Science Foundation (NSF) and EPSRC Materials World Network (MWN) grant (DMR 1210304 and EP/J018589/1). This work partially supported by the Cornell Center for Materials Research and made use of the Shared Facilities, which are supported through the NSF MRSEC program (DMR-1719875). We thank the Wiesner group at Cornell for the shared use of their AFM. SPA acknowledges a five-year ERC Advanced Investigator grant (PISA 320372) and EPSRC support (EP/J018589/1).

\section{REFERENCES}

(1) Weber, E.; Pokroy, B. Intracrystalline Inclusions within Single Crystalline Hosts: From Biomineralization to Bio-Inspired Crystal Growth. CrystEngComm 2015, 17, 5873-5883.

(2) Addadi, L.; Weiner, S. Biomineralization: Mineral Formation by Organisms. Phys. Scr. 2014, 89, 098003.

(3) Weiner, S.; Addadi, L. Crystallization Pathways in Biomineralization. Annu. Rev. Mater. Res. 2011, 41, 21-40.

(4) Kim, Y. Y.; Carloni, J. D.; Demarchi, B.; Sparks, D.; Reid, D. G.; Kunitake, M. E.; Tang, C. C.; Duer, M. J.; Freeman, C. L.; Pokroy, B.; Penkman, K., Harding J.H., Estroff, L.A., Baker, S.P., Meldrum, F.C. Tuning Hardness in Calcite by Incorporation of Amino Acids. Nat. Mater. 2016, 15, 903-910.

(5) Orme, C. A.; Noy, A.; Wierzbicki, A.; McBride, M. T.; Grantham, M.; Teng, H. H.; Dove, P. M.; DeYoreo, J. J. Formation of Chiral Morphologies through Selective Binding of Amino Acids to Calcite Surface Steps. Nature 2001, 411, 775-779.

(6) Davis, K. J.; Dove, P. M.; De Yoreo, J. J. The Role of $\mathrm{Mg}^{2+}$ as an Impurity in Calcite Growth. Science. 2000, 290, 1134-1137.

(7) Qiu, S. R.; Orme, C. A. Dynamics of Biomineral Formation at the near Molecular Level. Chem. Rev. 2008, 108, 4784-4822.

(8) Poloni, L. N.; Ford, A. P.; Ward, M. D. Site Discrimination and Anisotropic Growth Inhibition by Molecular Imposters on Highly Dissymmetric Crystal Surfaces. Cryst. Growth Des. 2016, 16, 5525-5541. 
(9) Rimer, J. D.; An, Z.; Zhu, Z.; Lee, M. H.; Goldfarb, D. S.; Wesson, J. A.; Ward, M. D. Crystal Growth Inhibitors for the Prevention of L-Cystine Kidney Stones through Molecular Design. Science 2010, 330, 337-341.

(10) Vekilov, P. G.; Chung, S.; Olafson, K. N. Shape Change in Crystallization of Biological Macromolecules. MRS Bull. 2016, 41, 375-380.

(11) Qiu, S. R.; Wierzbicki, A.; Orme, C. A.; Cody, A. M.; Hoyer, J. R.; Nancollas, G. H.; Zepeda, S.; De Yoreo, J. J. Molecular Modulation of Calcium Oxalate Crystallization by Osteopontin and Citrate. Proc. Natl. Acad. Sci. U. S. A. 2004, 101, 1811-1815.

(12) Cho, K. R.; Kim, Y.-Y.; Yang, P.; Cai, W.; Pan, H.; Kulak, A. N.; Lau, J. L.; Kulshreshtha, P.; Armes, S. P.; Meldrum, F. C.; De Yoreo, J.J. Direct Observation of Mineral-Organic Composite Formation Reveals Occlusion Mechanism. Nat. Commun. 2016, 7, 10187.

(13) Friddle, R. W.; Weaver, M. L.; Qiu, S. R.; Wierzbicki, A.; Casey, W. H.; De Yoreo, J. J. Subnanometer Atomic Force Microscopy of Peptide-Mineral Interactions Links Clustering and Competition to Acceleration and Catastrophe. Proc. Natl. Acad. Sci. U. S. A. 2010, 107, 11-15.

(14) Elhadj, S.; Salter, E. A.; Wierzbicki, A.; De Yoreo, J. J.; Han, N.; Dove, P. M. Peptide Controls on Calcite Mineralization: Polyaspartate Chain Length Affects Growth Kinetics and Acts as a Stereochemical Switch on Morphology. Cryst. Growth Des. 2006, 6, 197201.

(15) Elhadj, S.; De Yoreo, J. J.; Hoyer, J. R.; Dove, P. M. Role of Molecular Charge and Hydrophilicity in Regulating the Kinetics of Crystal Growth. Proc. Natl. Acad. Sci. U. S. A. 2006, 103, 19237-19242.

(16) Weaver, M. L.; Qiu, S. R.; Friddle, R. W.; Casey, W. H.; De Yoreo, J. J. How the Overlapping Timescales for Peptide Binding and Terrace Exposure Lead to Non-Linear Step Dynamics during Growth of Calcium Oxalate Monohydrate. Cryst. Growth Des. 2010, 10, 2954-2959.

(17) Shtukenberg, A. G.; Tripathi, K.; Ketchum, R.; Jeon, J. J.; Sanda, A.; Kahr, B. Incorporation of Macromolecules into $\alpha$-Lactose Monohydrate Crystals. Cryst. Growth Des. 2016, 16, 4589-4598.

(18) Li, H.; Xin, H. L.; Kunitake, M. E.; Keene, E. C.; Muller, D. A.; Estroff, L. A. Calcite Prisms from Mollusk Shells (Atrina Rigida): Swiss-Cheese-like Organic-Inorganic SingleCrystal Composites. Adv. Funct. Mater. 2011, 21, 2028-2034.

(19) Pokroy, B.; Fitch, A. N.; Marin, F.; Kapon, M.; Adir, N.; Zolotoyabko, E. Anisotropic Lattice Distortions in Biogenic Calcite Induced by Intra-Crystalline Organic Molecules. $J$. Struct. Biol. 2006, 155, 96-103.

(20) Kunitake, M. E.; Mangano, L. M.; Peloquin, J. M.; Baker, S. P.; Estroff, L. A. Evaluation of Strengthening Mechanisms in Calcite Single Crystals from Mollusk Shells. Acta Biomater. 2013, 9, 5353-5359.

(21) Chen, C.-L.; Qi, J.; Zuckermann, R. N.; DeYoreo, J. J. Engineered Biomimetic Polymers as Tunable Agents for Controlling $\mathrm{CaCO}_{3}$ Mineralization. J. Am. Chem. Soc. 2011, 133, 5214-5217.

(22) Ning, Y.; Fielding, L. A.; Doncom, K. E. B.; Penfold, N. J. W.; Kulak, A. N.; Matsuoka, H.; Armes, S. P. Incorporating Diblock Copolymer Nanoparticles into Calcite Crystals: Do Anionic Carboxylate Groups Alone Ensure Efficient Occlusion? ACS Macro Lett. 
2016, $5,311-315$.

(23) Kim, Y.-Y.; Ribeiro, L.; Maillot, F.; Ward, O.; Eichhorn, S. J.; Meldrum, F. C. BioInspired Synthesis and Mechanical Properties of Calcite-Polymer Particle Composites. Adv. Mater. 2010, 22, 2082-2086.

(24) Kim, Y. Y.; Ganesan, K.; Yang, P.; Kulak, A. N.; Borukhin, S.; Pechook, S.; Ribeiro, L.; Kröger, R.; Eichhorn, S. J.; Armes, S. P.; Pokroy, B., Meldrum, F.C. An Artificial Biomineral Formed by Incorporation of Copolymer Micelles in Calcite Crystals. Nat. Mater. 2011, 10, 890-896.

(25) Muñoz-Espí, R.; Qi, Y.; Lieberwirth, I.; Gómez, C. M.; Wegner, G. SurfaceFunctionalized Latex Particles as Controlling Agents for the Mineralization of Zinc Oxide in Aqueous Medium. Chem. - A Eur. J. 2006, 12, 118-129.

(26) Wegner, G.; Demir, M. M.; Faatz, M.; Gorna, K.; Munoz-Espi, R.; Guillemet, B.; Gröhn, F. Polymers and Inorganics: A Happy Marriage? Macromol. Res. 2007, 15, 95-99.

(27) Kim, Y.-Y.; Semsarilar, M.; Carloni, J. D.; Cho, K. R.; Kulak, A. N.; Polishchuk, I.; Hendley, C. T.; Smeets, P. J. M.; Fielding, L. A.; Pokroy, B.; Tang, C.C., Estroff, L.A., Baker, S.B., Armes, S.P., Meldrum, F.C. Structure and Properties of Nanocomposites Formed by the Occlusion of Block Copolymer Worms and Vesicles Within Calcite Crystals. Adv. Funct. Mater. 2016, 26, 1382-1392.

(28) Ning, Y.; Fielding, L. A.; Ratcliffe, L. P. D.; Wang, Y.-W.; Meldrum, F. C.; Armes, S. P. Occlusion of Sulfate-Based Diblock Copolymer Nanoparticles within Calcite: Effect of Varying the Surface Density of Anionic Stabilizer Chains. J. Am. Chem. Soc. 2016, 138, 11734-11742.

(29) Canning, S. L.; Smith, G. N.; Armes, S. P. A Critical Appraisal of RAFT-Mediated Polymerization-Induced Self-Assembly. Macromolecules 2016, 49, 1985-2001.

(30) Warren, N. J.; Armes, S. P. Polymerization-Induced Self-Assembly of Block Copolymer Nano-Objects via RAFT Aqueous Dispersion Polymerization. J. Am. Chem. Soc. 2014, 136, 10174-10185.

(31) Derry, M. J.; Fielding, L. A.; Armes, S. P. Polymerization-Induced Self-Assembly of Block Copolymer Nanoparticles via RAFT Non-Aqueous Dispersion Polymerization. Prog. Polym. Sci. 2016, 52, 1-18.

(32) Hanisch, A.; Yang, P.; Kulak, A. N.; Fielding, L. A.; Meldrum, F. C.; Armes, S. P. Phosphonic Acid-Functionalized Diblock Copolymer Nano-Objects via PolymerizationInduced Self-Assembly: Synthesis, Characterization, and Occlusion into Calcite Crystals. Macromolecules 2016, 49, 192-204.

(33) Ning, Y.; Fielding, L. A.; Andrews, T. S.; Growney, D. J.; Armes, S. P. Sulfate-Based Anionic Diblock Copolymer Nanoparticles for Efficient Occlusion within Zinc Oxide. Nanoscale 2015, 7, 6691-6702.

(34) Munk, P. Equilibrium and Nonequilibrium Polymer Micelles. In Solvents and SelfOrganization of Polymers; Springer Netherlands: Dordrecht, 1996; pp 19-32.

(35) Lovett, J. R.; Warren, N. J.; Ratcliffe, L. P. D.; Kocik, M. K.; Armes, S. P. pH-Responsive Non-Ionic Diblock Copolymers: Ionization of Carboxylic Acid End-Groups Induces an Order-Order Morphological Transition. Angew. Chemie - Int. Ed. 2015, 54, 1279-1283.

(36) Semsarilar, M.; Jones, E. R.; Blanazs, A.; Armes, S. P. Efficient Synthesis of StericallyStabilized Nano-Objects via RAFT Dispersion Polymerization of Benzyl Methacrylate in 
Alcoholic Media. Adv. Mater. 2012, 24, 3378-3382.

(37) Semsarilar, M.; Ladmiral, V.; Blanazs, A.; Armes, S. P. Poly(Methacrylic Acid)-Based $\mathrm{AB}$ and $\mathrm{ABC}$ Block Copolymer Nano-Objects Prepared via RAFT Alcoholic Dispersion Polymerization. Polym. Chem. 2014, 5, 3466-3475.

(38) Parnell, A. J.; Martin, S. J.; Dang, C. C.; Geoghegan, M.; Jones, R. A. L.; Crook, C. J.; Howse, J. R.; Ryan, A. J. Synthesis, Characterization and Swelling Behaviour of Poly(Methacrylic Acid) Brushes Synthesized Using Atom Transfer Radical Polymerization. Polymer 2009, 50, 1005-1014.

(39) Minko, S. Responsive Polymer Brushes. J. Macromol. Sci. Part C Polym. Rev. 2006, 46, 397-420.

(40) Berman, A.; Addadi, L.; Kvick, A.; Leiserowitz, L.; Nelson, M.; Weiner, S. Intercalation of Sea Urchin Proteins in Calcite: Study of a Crystalline Composite Material. Science 1990, 250, 664-667.

(41) Metzler, R. A.; Tribello, G. A.; Parrinello, M.; Gilbert, P. U. P. A. Asprich Peptides Are Occluded in Calcite and Permanently Disorder Biomineral Crystals. J. Am. Chem. Soc. 2010, 132, 11585-11591.

(42) Meldrum, F. C.; Hyde, S. T. Morphological Influence of Magnesium and Organic Additives on the Precipitation of Calcite. J. Cryst. Growth 2001, 231, 544-558.

(43) Poloni, L. N.; Zhong, X.; Ward, M. D.; Mandal, T. Best Practices for Real-Time in Situ Atomic Force and Chemical Force Microscopy of Crystals. Chem. Mater. 2017, 29, 331345.

(44) De Yoreo, J. J.; Chung, S.; Friddle, R. W. In Situ Atomic Force Microscopy as a Tool for Investigating Interactions and Assembly Dynamics in Biomolecular and Biomineral Systems. Adv. Funct. Mater. 2013, 23, 2525-2538.

(45) De Yoreo, J. J.; Vekilov, P. G. Principles of Crystal Nucleation and Growth. Rev. Mineral. Geochemistry 2003, 54, 57-93.

(46) De La Pierre, M.; Raiteri, P.; Gale, J. D. Structure and Dynamics of Water at Step Edges on the Calcite $\{10 \overline{14}\}$ Surface. Cryst. Growth Des. 2016, 16, 5907-5914.

(47) De La Pierre, M.; Raiteri, P.; Stack, A. G.; Gale, J. D. Uncovering the Atomistic Mechanism for Calcite Step Growth. Angew. Chem. Int. Ed. 2017, 56, 8464-8467.

(48) Derry, M. J.; Fielding, L. A.; Armes, S. P. Industrially-Relevant Polymerization-Induced Self-Assembly Formulations in Non-Polar Solvents: RAFT Dispersion Polymerization of Benzyl Methacrylate. Polym. Chem. 2015, 6, 3054-3062.

(49) Aizenberg, J.; Black, A. J.; Whitesides, G. M. Control of Crystal Nucleation by Patterned Self-Assembled Monolayers. Nature 1999, 398, 495-498. 


\section{Graphical TOC}

\section{For Table of Contents use only}

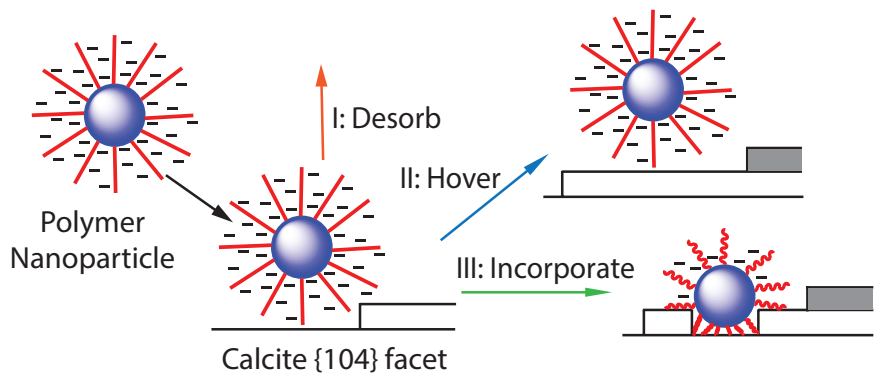

